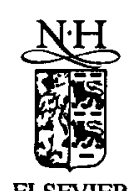

ELSEVIER

Nuclear Physics B 502 (1997) 125-148

\title{
Brane configurations and 4D field theory dualities
}

\author{
A. Brandhuber ${ }^{\mathrm{a}, 1}$, J. Sonnenschein ${ }^{\mathrm{a}, 1}$, S. Theisen ${ }^{\mathrm{b}, 2}$, S. Yankielowicz ${ }^{\mathrm{a}, 1}$ \\ ${ }^{a}$ School of Physics and Astronomy, Beverly and Raymond-Sackler Faculty of Exact Sciences, Tel-Aviv \\ University, Ramat-Aviv, Tel-Aviv 69978, Israel \\ b Sektion Physik, Universität München, Theresienstraße 37, 80333 Munich, Germany
}

Received 28 April 1997; accepted 17 June 1997

\begin{abstract}
We study brane configurations which correspond to field theories in four dimension with $N=2$ and $N=1$ supersymmetry. In particular we discuss brane motions that translate to Seiberg's duality in $N=1$ models recently studied by Elitzur, Giveon and Kutasov. We investigate, using the brane picture, the moduli spaces of the dual theories. Deformations of these models like mass terms and vacuum expectation values of scalar fields can be identified with positions of branes. The map of these deformations between the electric and dual magnetic theories is clarified. The models we study reproduce known field theory results and we provide an example of new dual pairs with $N=1$ supersymmetry. Possible relations between brane configurations and non-supersymmetric field theories are discussed. (c) 1997 Published by Elsevier Science B.V.
\end{abstract}

\section{Introduction}

Recently, it has become clear that many of the dualities and exact results in supersymmetric field theories have direct realizations in terms of string theory. The main idea is to construct a local description of the field theory in a string theory setup. This local description involves geometric aspects of the compactification manifold [1,2], the local geometry together with D-branes wrapped around homology cycles [3]. The simplest approach, however, is the one taken in [4], where one finds an arrangement of flat Dand NS branes in $d=1$ Minkowski space, such that the field theory on the world-

\footnotetext{
${ }^{1}$ Work supported in part by the US-Israel Binational Science Foundation, by GIF - the German-Israeli Foundation for Scientific Research, and by the Israel Science Foundation.

${ }^{2}$ Work supported in part by GIF - the German-Israeli Foundation for Scientific Research and by the European Commission TMR programme ERBFMRX-CT96-0045, in which S.T. is associated to HU-Berlin.
} 
volume of the D-branes has the desired gauge symmetry, matter content and number of supersymmetries.

In [4] Dirichlet threebranes ending on Dirichlet fivebranes or solitonic fivebranes in IIB string theory were used to study field theories in three dimensions. The fivebranes are heavier than the threebranes and can be treated classically whereas the worldvolume of the threebrane is finite in one direction. Thus, at low energies the threebrane world-volume appears to be three-dimensional. The world-volume theory of threebranes stretched between two solitonic branes has $N=4$ supersymmetry in three dimensions and the inclusion of Dirichlet fivebranes leads to matter multiplets in the world-volume theory coming from open strings between the Dirichlet fivebranes and the Dirichlet threebranes. Brane configurations corresponding to field theories in three dimensions with $N=4$ were also studied in $[5,6]$. Threebranes stretched between two different types of solitonic fivebranes which lead to field theories with $N=2$ supersymmetry were investigated in [5].

A rather similar construction in IIA string theory was introduced by Elitzur, Giveon and Kutasov [7] to analyze four-dimensional theories with $U(n)$ gauge groups. The generalization to $S O(N)$ and $U S p(N)$ gauge groups can be found in [8]. In this case Dirichlet fourbranes stretched between two types of solitonic fivebranes lead, at low energy, to an effective four dimensional world-volume field theory with $N=1$ supersymmetry since one of the fourbrane directions is finite. The addition of Dirichlet sixbranes (replacing the Dirichlet fivebranes in IIB) corresponds to additional chiral matter multiplets. By making certain deformations in the brane configuration Seiberg's duality [9] and the duality of theories with adjoint matter [10] can be realized.

The aim of this paper is to study in general brane configurations in IIA which correspond to field theories in four dimensions with $N=2, N=1$ and possibly $N=0$ supersymmetry. In particular using brane rearrangements we provide further evidence for

(i) the equivalence between the Higgs branches of "dual" $N=2$ models,

(ii) the correspondence between the deformations of the $N=1$ electric and magnetic theories associated with Seiberg's duality,

(iii) generalization of the latter theories to theories with product gauge groups.

In Section 2 we explain the basic types of brane configurations in type IIA string theory used in this paper and treat other general aspects as the classical global symmetries of the world-volume theories and their partial breaking by instantons, and creation/annihilation processes of fourbranes. In Section 3, we analyze models with $N=2$ supersymmetry and provide a brane description of the duality in the Higgs branches of these theories. In Section 4, we study $N=1$ supersymmetric theories. We review the brane description of Seiberg's duality and adjoint duality [7] and precisely identify deformations of the theories and work out the map between the deformations in the electric theories and the dual magnetic theories. Furthermore we study a configuration with three solitonic fivebranes which provides a new dual pair of theories with product gauge groups. In Section 5, we try to make contact with dualities in gauge theories without any supersymmetry. We introduce a set of branes that breaks all 32 supersym- 


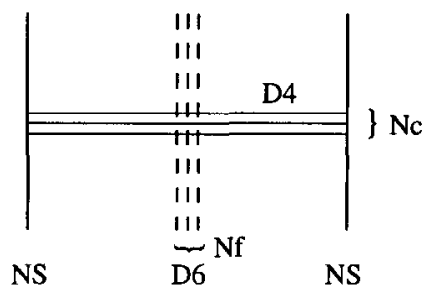

Fig. 1.

metries of IIA. We analyze the possibility for having brane configurations which may be related to duality in non-supersymmetric QCD with adjoint fermions. In Section 6, we address the problem of identifying field theory instantons in the brane setup.

\section{From brane configurations to field theory - generalities}

We will use the same types of branes in type IIA string theory which were introduced in [7]: two types of Neveu-Schwarz five branes NS and $\mathrm{NS}^{\prime}$ whose world-volumes have coordinates $x^{0}, x^{1}, x^{2}, x^{3}, x^{4}, x^{5}$ and $x^{0}, x^{1}, x^{2}, x^{3}, x^{8}, x^{9}$, respectively, a Dirichlet sixbrane D6 whose world-volume has coordinates $x^{0}, x^{1}, x^{2}, x^{3}, x^{7}, x^{8}, x^{9}$ and a Dirichlet fourbrane D4 with world-volume $x^{0}, x^{1}, x^{2}, x^{3}, x^{6}$. This set of branes preserves $1 / 8$ of the 32 supercharges of the type IIA string theory. A $T$-duality transformation in the $x^{3}$ direction reproduces the configuration in IIB theory recently studied in [5]. Note that configurations without the NS ${ }^{\prime}$ type of branes break only 3/4 of the supersymmetry of the original theory and $T$-duality in the $x^{3}$ direction gives the configurations studied in [4] which correspond to $3 \mathrm{~d}$ gauge field theories with $N=4$ supersymmetry.

$N=2$ configurations $($ cf. Fig. 1 )

We start with the simplest configuration involving two NS branes at equal position in the $x^{7}, x^{8}, x^{9}$ directions and at different locations in the $x^{6}$ direction. Between the two NS branes $N_{c}$ D4-branes are stretched. Their location in the $x^{7}, x^{8}, x^{9}$ directions is fixed and their world-volume is $\mathbb{R}^{1,3}$ times a finite interval in the $x_{\mathrm{NS}}^{6}<x^{6}<x_{\mathrm{NS}^{\prime}}^{6}$ direction. The world-volume theory on the D4-branes at long distances (compared to the $x^{6}$ direction) is a four-dimensional $N=2$ supersymmetric Yang-Mills theory with gauge group $U\left(N_{c}\right)$. The $N=2$ vector multiplet consists of a $N=1$ vector multiplet $W_{\alpha}$ which is associated with open strings between the $N_{c}$ D4-branes themselves and an adjoint $N=1$ chiral multiplet $\Phi$, whose two real scalar components correspond to fluctuations in the $x^{4}, x^{5}$ directions. Separating the D4-branes in the $x^{4}, x^{5}$ directions corresponds to going to a Coulomb branch of the $N=2$ theory.

If we put a set of $N_{f}$ D6-branes at definite values of $x^{4}, x^{5}, x^{6}$ there are $N_{f}$ hypermultiplets of $N=2$ supersymmetry (or $N_{f}$ pairs of chiral multiplets) in the fundamental representation of the gauge group $U\left(N_{c}\right)$ ) coming from strings between the D4- and D6-branes. Recall that a hypermultiplet is equivalent to a pair of chiral multiplets $Q$ and $\tilde{Q}$ in $N=1$ language transforming in the fundamental and the anti-fundamental representation of the gauge group, respectively. 


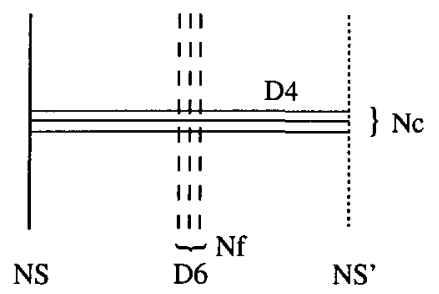

Fig. 2 .

$N=1$ configurations (cf. Fig. 2)

Another possibility is a configuration with one NS and one $\mathrm{NS}^{\prime}$ at definite values of $x^{6}, x^{7}, x^{8}, x^{9}$ and $x^{4}, x^{5}, x^{6}, x^{7}$, respectively, separated in the $x^{6}$ direction. The difference from the $N=2$ configuration is that the positions of the $N_{c}$ D4-branes are now completely fixed. We cannot separate the D4-branes and there is no adjoint chiral field. The world-volume theory on the D4-branes at long distances is a $N=1$ supersymmetric Yang-Mills theory with gauge group $U\left(N_{c}\right)$ [7].

If we put a set of $N_{f}$ D6-branes at definite values of $x^{4}, x^{5}, x^{6}$ there are $N_{f}$ pairs of chiral (quark) multiplets $Q$ and $\tilde{Q}$ in the (anti-)fundamental representation of the gauge group $U\left(N_{c}\right)$ due to strings between the D4- and D6-branes [7].

In Section 4.3 we discuss generalization of this configuration to a model with a product gauge group. For both, the $N=2$ and $N=1$ configurations, moving the D6branes away from the $\mathrm{D} 4$-branes in the $x^{4}, x^{5}$ directions corresponds to mass terms for the quarks in the world-volume theory which are of the form $m Q \tilde{Q}$.

\section{$N=0$ configurations}

Brane configurations which may be relevant to the study of field theories without supersymmetry will be the subject of Section 5 .

\section{Global symmetries}

We introduced $N_{f}$ D6-branes which produced matter multiplets on the D4 brane world-volume theory corresponding to strings between the D4- and D6-branes. But there are also strings between the D6-branes themselves which give rise to an $U\left(N_{f}\right)$ gauge theory on the D6 world-volume. Since the D6-branes are much heavier than the D4-branes (they extend in two additional dimensions) to an D4-brane observer the $U\left(N_{f}\right)$ gauge theory on the D6-branes appears as a global symmetry - the $U\left(N_{f}\right)$ flavor symmetry. The $U(1)$ subgroup of the $U\left(N_{f}\right)$ flavor group is the $U(1)_{B}$ symmetry associated with the conservation of baryon number. In the corresponding field theory the actual classical flavor symmetry of the massless $N=1$ theory is $U\left(N_{f}\right) \times U\left(N_{f}\right)$. In the $N=2$ case this symmetry is broken explicitly by the $N=2$ superpotential to the diagonal $U\left(N_{f}\right)$ symmetry.

The $N=1$ and the $N=2$ configurations are invariant under rotations $S O(2)_{45} \sim$ $U(1)_{45}$ in the $x^{4}, x^{5}$ plane which corresponds to the $U(1)_{R}$ symmetry. The $N=2$ configurations have a rotation symmetry $S O(3)_{789}$ in the $x^{7}, x^{8}, x^{9}$ directions whose double cover corresponds to the $S U(2)_{R}$ symmetry of the $N=2$ supersymmetry algebra. 
The $N=1$ configurations are invariant only under its Abelian subgroup $U(1)_{89}$ which we will denote as the $U(1)_{J}$ symmetry. (This $U(1)$ symmetry is also present in certain brane configurations without supersymmetry which are discussed in Section 5).

To summarize we list the fields and parameters with their transformation properties. ${ }^{3}$ $M, q$ and $\tilde{q}$ are the meson and the (anti-) quark fields, respectively, that will appear in later sections in the magnetic dual theories:

$\begin{array}{lccc} & U(1)_{45}=U(1)_{R} & U(1)_{89}=U(1)_{J} & U(1)_{B} \\ W_{\alpha} & 1 & 1 & 0 \\ \Phi & 2 & 0 & 0 \\ Q, \tilde{Q} & 0 & 1 & R_{Q},-R_{Q} \\ q, \tilde{q} & 0 & 1 & R_{q},-R_{q} \\ M & 2 & 0 & 0 \\ m & 2 & 0 & 0\end{array}$

Note that the values of the quantum numbers with respect to $U(1)_{R}$ and $U(1)_{J}$ are only the classical values. In the quantum theory the charges are different and only a combination of the two $U(1)$ symmetries remains unbroken. This means that string loop/instanton effects should break the $U(1)_{45} \times U(1)_{89}$ to a single unbroken $U(1)_{\mathcal{R}}$. The quantum mechanical assignments of the various charges will be given in a particular case in Section 4.3.

\section{Creation and annihilation of D4-branes}

Whenever a D6-brane moves in the $x^{6}$ direction towards an NS brane and passes through it a new D4-brane stretching between the NS and the D6-brane is created [7]. Note that the NS and D6-brane cannot be separated in any other direction. In contrast a NS ${ }^{\prime}$ and a D6-brane can avoid each other in the $x^{4}, x^{5}$ directions. Such a process was first studied in [4] in type IIB string theory. A reversed process in which a D4-brane stretched between a NS and a D6-brane is annihilated; we will encounter it later.

In $[4]$ the nature of this creation/annihilation process was made more precise using linking numbers assigned to each brane. The total linking number of each five-brane that is invariant under any move is the total magnetic charge measured on that five-brane. It is defined as the sum of the linking number and the number of D4-branes with given orientation ending on it. A D4-brane boundary looks like a magnetic charge on the D6brane. This can be seen using the results of [11] where it was shown that in IIB string theory a D string can end on a D3-brane and that the endpoint of the D string on the D3-brane is a magnetically charged particle. $T$-dualizing in three transverse directions we get a configuration of a D4-brane ending on a D6-brane in IIA string theory. If a D6-brane moves through a NS brane its linking number changes by one unit and consequently a D4-brane is created leaving the total magnetic charge invariant. The total magnetic charge measured on a D6 and a NS brane is [4]

\footnotetext{
${ }^{3}$ For example, the mass parameter $m$ corresponds to moving the D6 in the $x^{4}, x^{5}$ directions. Therefore it transforms as a vector of $S O(2)_{45} \sim U(1)_{45}$ and we assign charge 2 to it. Spinors of $U(1)_{45}$ have charge 1.
} 


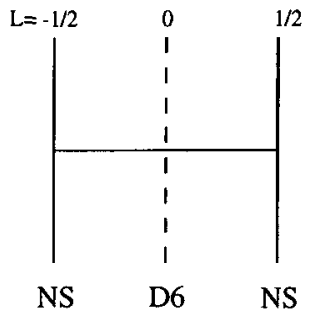

(a)

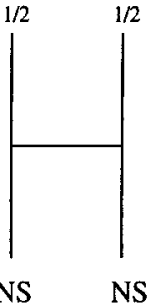

(b)

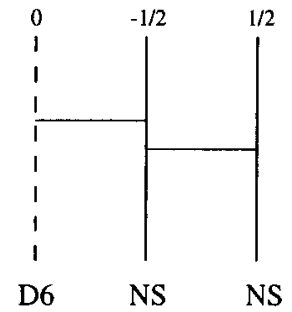

(c)

Fig. 3.

$$
\begin{aligned}
& L_{\mathrm{NS}}=\frac{1}{2}\left(r_{\mathrm{D} 6}-l_{\mathrm{D} 6}\right)+(L-R), \\
& L_{\mathrm{D} 6}=\frac{1}{2}\left(r_{\mathrm{NS}}-l_{\mathrm{NS}}\right)+(L-R),
\end{aligned}
$$

where $r$ and $l$ denote the number of branes of the specified type on the right and on the left of the brane, $L$ and $R$ is the number of D4-branes ending to the left and to the right. The above expressions are not modified by the presence of $\mathrm{NS}^{\prime}$ branes.

In Fig. 3 an example of a creation process is depicted. We have indicated the total magnetic charges on top of the branes. The starting configuration of Fig. 3a consists of two NS branes connected by a D4-brane, and a D6-brane between the two NS branes. This corresponds to an $U(1) N=2$ supersymmetric gauge theory with one charged hypermultiplet which comes from an open string between the D4- and the D6-brane. If we move D6 through the left NS brane we would naively expect to get the configuration shown in Fig. 3b. This corresponds to a $U(1)$ theory without hypermultiplet. A careful analysis à la [4] shows that the final configuration must be as in Fig. 3c where a D4brane between the left NS and the D6-brane is created in the transition. We obtain the same theory on the D4-brane world-volume although the hypermultiplet now corresponds to an open string between the left and the right D4-brane. A geometrical interpretation of this process has recently been given in [12].

\section{3. $N=2$ gauge theories and branes}

We start with a configurations of D6-, D4- and NS branes. Recall that in this case only $3 / 4$ of the supersymmetry is broken and there is $N=2$ supersymmetry on the D4-brane world-volume. We start with a configuration of two NS branes and $N_{c}$ D4branes stretched between them. The world-volume theory on the D4-branes is $N=2$ supersymmetric Yang-Mills with gauge group $U\left(N_{c}\right)$. The D4-branes reside at definite values of $x^{7}, x^{8}, x^{9}$ but their positions in the $x^{4}, x^{5}$ directions are allowed to fluctuate. This fluctuation corresponds to the adjoint (complex) scalar of the $N=1$ chiral multiplet $\Phi$. If we move the D4-branes in these directions we give vevs to the complex scalar field and we are in the Coulomb branch of the $N=2$ gauge theory. As discussed in the previous section this configuration is symmetric under rotations in the $x^{4}, x^{5}$ directions $U(1)_{45}=U(1)_{R}$. In the quantum field theory this symmetry is broken by instantons to 


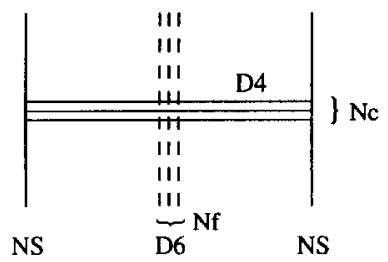

(a)

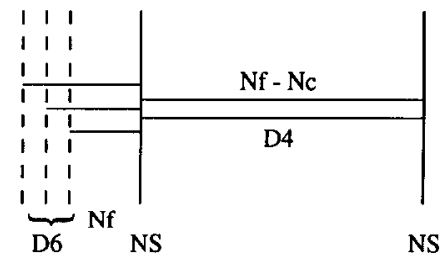

(b)

Fig. 4.

some discrete subgroup. The $S U(2)_{R}$ symmetry of the $N=2$ algebra comes from the rotational invariance in the $x^{7}, x^{8}, x^{9}$ directions.

By putting $N_{f}$ D6-branes between the two NS branes we can add $N_{f}$ hypermultiplets $H_{N=2}=(Q, \tilde{Q})_{N=1}$ in the fundamental representation of the gauge group.

Moving the D6-branes in the $x^{4}, x^{5}$ directions corresponds to mass terms for the hypermultiplets $H$. Furthermore if more than one D6-brane touches a D4-brane the D4-brane splits into pieces which can move between the D6-branes independently in the $x^{7}, x^{8}, x^{9}$ directions. This corresponds to giving a vev to a quark where the vev is proportional to the separation. The fourth scalar in the $N=2$ hypermultiplet corresponds to a Wilson line in the $x^{6}$ direction, namely the $A_{6}$ component of the gauge field on the D4-brane. This reduces, in the field theory picture, the $U\left(N_{c}\right) \times S U\left(N_{f}\right)$ group to $U\left(N_{c}-1\right) \times S U\left(N_{f}-1\right)$.

Wc now want to establish the duality of the Higgs branches of the theory with gauge group $U\left(N_{c}\right)$ and $N_{f}$ flavors and the theory with dual gauge group $U\left(N_{f}-N_{c}\right)$ and $N_{f}$ flavors [13]. For that purpose we perform certain moves in the brane configuration which are the same as in [7].

(i) The starting configuration (Fig. 4a) consists of two NS branes separated in the $x^{6}$ direction, $N_{c}$ D4-branes stretched between them with $x^{4}=x^{5}=0$ (i.e. the vev of the scalar field in the vectormultiplet is zero) and $N_{f}$ D6-branes which touch the D4-branes $x^{4}=x^{5}=0$ (the mass terms are zero since we want to study the Higgs phase). We move the D6-branes to the left, crossing the left NS brane. In this process we create $N_{f}$ D4-branes stretched between the D6-branes and the left NS brane.

(ii) Out of these D4-branes $N_{c}$ can connect with the D4-branes stretched between the two NS branes (this requires that $N_{f} \geqslant N_{c}$ ) leaving us with $N_{c}$ D4-branes between the D6-brane and the right NS, and $N_{f}-N_{c}$ D4-branes stretched between the D6 and the left NS brane. The left NS brane can now move in the $x^{7}, x^{8}, x^{9}$ direction relative to the right NS brane.

(iii) The left NS brane moves in the $x^{6}$ direction to the other side of the right NS brane without meeting in space-time. Nothing special happens in this step.

(iv) The NS brane comes back to its original position in the $x^{7}, x^{8}, x^{9}$ directions, the $N_{f}-N_{c}$ D4-branes connecting to $N_{f}-N_{c}$ D6-branes touch the second NS brane and split into $N_{f}-N_{c}$ D4-branes stretched between the two NS branes and $N_{f}-N_{c}$ D4-branes stretched between $N_{f}-N_{c}$ D6-branes and the NS brane (Fig. 4b).

In the final configuration there are $N_{f}$ D4-branes stretched between the $N_{f}$ D6- 


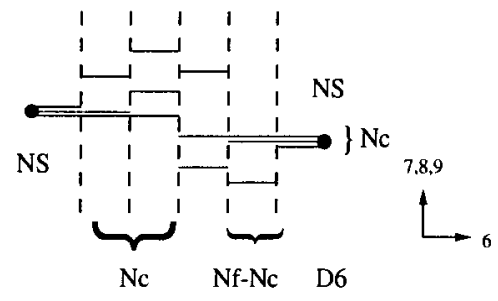

Fig. 5.

branes and a NS brane, and $N_{f}-N_{c}$ D4-branes connecting the two NS branes. The world-volume theory on the D4-branes is a $N=2 U\left(N_{f}-N_{c}\right)$ gauge theory with $N_{f}$ hypermultiplets from open strings connecting the two sets of D4-branes. Since the positions of the $N_{f}$ D4-branes between the D6-branes and the NS brane are completely fixed there are no massless states coming from open strings between these D4-branes (no mesons), as expected from field theory.

There is an alternative way to see that the NS branes can move in the $x^{7}, x^{8}, x^{9}$ directions which corresponds to a FI term in the field theory. Remember that this feature is crucial to make all the moves to obtain the dual brane configuration. The main point is to split the D4-branes as much as possible already in the starting position, i.e. we choose a generic point in the Higgs moduli space. In order to do so the D6-branes must touch the D4-branes which allows the D4-branes to split and the parts between two D6-branes can move in the $x^{7}, x^{8}, x^{9}$ directions. Note that there is a subtlety for D4-branes stretched between an NS and an D6-brane which is explained in the next paragraph. Only if $N_{f} \geqslant N_{c}$ can all the D4-branes split and the two NS branes are free to move with respect to each other (together with the D4-branes attached to them) in the $x^{7}, x^{8}, x^{9}$ directions (Fig. 5). The relative offset between the two NS branes corresponds to turning on FI terms in the field theory on the brane. On the Higgs branch FI terms do not break supersymmetry.

Let us end this section with a comment on the dimension of the Higgs branch. To count the number of parameters we split the D4-branes between the D6-branes as much as possible. Remember that the D4-brane stretched between an NS and a D6-brane cannot move whereas a D4-brane between two D6-branes can move. Each D4-brane which is allowed to move gives two complex parameters (the $x^{7}, x^{8}, x^{9}$ directions and a Wilson line in the $x^{6}$ direction). The naive counting would then give $2 N_{c}\left(N_{f}-1\right)$ which contradicts our expectation from field theory where the dimension is $2 N_{c}\left(N_{f}-N_{c}\right)$. To remedy this situation recall the discussion of the so called $s$-configurations in [4]. The main point is that if we have a NS and a D6-brane we get a supersymmetric vacuum only if there is just one D4-brane stretched between them. So we have to be more careful in the splitting of the D4-branes and we have to avoid such s-configurations. The resulting picture is given in Fig. 5 and the counting now gives the correct dimension of the Higgs branch $2 N_{c}\left(N_{f}-N_{c}\right)$. This is invariant under $N_{c} \leftrightarrow N_{f}-N_{c}$. 


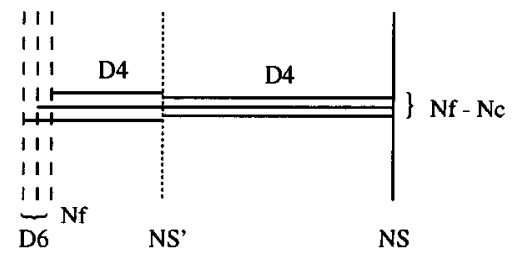

Fig. 6.

\section{Dualities in $N=1$ theories}

\subsection{Seiberg's duality}

The relevant configuration was proposed in [7] and is drawn in Fig. 2. The NS brane is located at $x^{6}=x_{\mathrm{NS}}^{6}<x_{\mathrm{NS}}^{6}$ and $x^{7}=x^{8}=x^{9}=0$, the NS $\mathrm{N}^{\prime}$ brane is at $x^{6}=x_{\mathrm{NS}^{\prime}}^{6}>x_{\mathrm{NS}}^{6}$ and $x^{4}=x^{5}=x^{7}=0$. The D4-branes reside at $x^{4}=x^{5}=x^{7}=x^{8}=x^{9}=0$ and are extended in the $x^{6}$ direction with $x_{\mathrm{NS}}^{6}<x^{6}<x_{\mathrm{NS}}^{6}$. For the moment the D6-branes are chosen to reside at $x^{4}=x^{5}=0$. We will discuss deformations of this configuration later. The $x^{6}$ positions of the D6-branes are somewhere between the NS and the NS' brane. This means that the D6-branes touch the D4-branes and the quarks which correspond to open strings between the D4- and the D6-branes are massless. As was shown in [7], to get to the magnetic description we have to go through the following four moves.

(i) We move all D6-branes to the left of the NS brane. When the $x^{6}$ positions of a D6 and a NS branc coincide they actually mect in the $x^{0}, x^{1}, x^{2}, x^{3}$ directions. By passing through the NS brane a D4-brane is generated for each D6 and this D4-brane is stretched between the NS and the D6-brane. The world-volume theory on the D4-branes does not change, only the (anti-) quarks correspond now to open strings between the two sets of D4-branes. Now $N_{c}$ D4-branes of the $N_{f}$ stretched between the D6-branes and the NS brane can be connected with the $N_{c}$ D4-branes stretched between the NS and the $\mathrm{NS}^{\prime}$ brane. For this it is important that $N_{f} \geqslant N_{c}$, otherwise there is always at least one D4-brane between NS and NS ${ }^{\prime}$ which would force the $x^{7}$ positions of the NS and the NS ${ }^{\prime}$ to be equal. Thus we have $N_{c}$ D4-branes between the D6 and the NS ${ }^{\prime}$, and $N_{f}-N_{c}$ D4-branes between the D6 and the NS.

(ii) Since none of the D4-branes ending on NS ${ }^{\prime}$ also ends on the NS, the NS brane can be lifted in the $x^{7}$ direction away from the NS'. This corresponds to turning on a Fayet-Iliopoulos $D$-term for the $U(1)$ factor of the gauge group. Since we have turned off the mass terms we are in the Higgs branch and in this case the FI $D$-term does not break supersymmetry.

(iii) Now the NS brane can move to the other side of the NS' brane such that they avoid each other. Nothing special happens in this step.

(iv) Next we turn off the FI $D$-term which means that the $x^{7}$ position of the NS brane returns to its original value. This implies that the $\mathrm{NS}^{\prime}$ touches the $N_{f}-N_{c}$ D4-branes and they can split. The final configuration is therefore $N_{f}$ D4-brane between the D6 and $\mathrm{NS}^{\prime}$ brane and $N_{f}-N_{c}$ D4-branes between NS and NS ${ }^{\prime}$ (Fig. 6). The matter content is equal to Seiberg's magnetic theory. The gauge group is $U\left(N_{f}-N_{c}\right)$, there are $N_{f}$ 


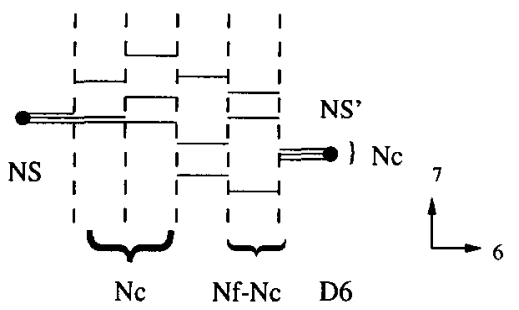

Fig. 7.

magnetic (anti-)quarks) in the (anti-)fundamental of the gauge group coming from strings between the two sets of D4-branes, and the magnetic mesons which arise from strings between the $N_{f}$ D4-branes between the D6-branes and the $\mathrm{NS}^{\prime}{ }^{4}$

To gain more insight into the issue of the FI term it is useful to start at a generic point of the Higgs branch. This means that in the starting position the D6-branes touch the D4branes which split into two parts. The position of the left part of a D4-brane is always fixed, but the right part can move in the $x^{8}, x^{9}$ directions. To avoid $s$-configurations only one D4-brane part is allowed to stretch between the NS and one specific D6-brane, but between two D6-branes there can be more than one D4-branes separated in the $x^{7}, x^{8}, x^{9}$ directions. There is also the possibility of D4-brane parts between D6-branes and the $\mathrm{NS}^{\prime}$ brane which can move in the $x^{8}, x^{9}$ directions. Complete splitting is only possible for $N_{f} \geqslant N_{c}$ and once this is achieved the NS brane can move together with the D4-branes attached to it in the $x^{7}, x^{8}, x^{9}$ directions (Fig. 7). Out of the three parameters only the separation of the NS and the $\mathrm{NS}^{\prime}$ in the $x^{7}$ direction corresponds to the FI term since the world-volume of the NS $S^{\prime}$ brane extends in the $x^{8}, x^{9}$ directions.

We now want to study in turn two different deformations of the theories in the electric and the magnetic description. We recall that the correspondence between the deformed clectric and magnetic theories formed the basis for the belief in the duality within the field theory context.

\section{Mass terms}

Giving a mass to one of the electric quarks reduces the flavor symmetry $U\left(N_{f}\right)$ to $U\left(N_{f}-1\right)$. The field theories on the D4-branes have a classical flavor group bigger than $U\left(N_{f}\right)$, namely $U\left(N_{f}\right) \times U\left(N_{f}\right)$ but in the brane context we see only the diagonal part of it which arises from the D6-brane sector. In the magnetic theory giving a mass to one of the mesons induces a non-zero vev for one of the magnetic quarks via the equations of motion of the massive meson. Thus the magnetic gauge group is reduced to $U\left(N_{f}-N_{c}-1\right)$. What does this correspond to in the brane configuration? The starting point is a configuration of one NS and one NS $S^{\prime}$ brane, $N_{c}$ D4-branes stretched between NS and NS ${ }^{\prime}$, all on top of each other and $N_{f}$ D6-branes. Moving one of the D6-branes in the $x^{4}, x^{5}$ directions away from the D4-branes (Fig. 8a) gives mass to a quark in

\footnotetext{
${ }^{4}$ Near the origin of the moduli space the correct identification of the mesons involves also mixing with states arising from strings between the $N_{f}$ D4-branes and the D6-branes. We will discuss this point later. We would like to thank $O$. Aharony for discussions on this issue.
} 

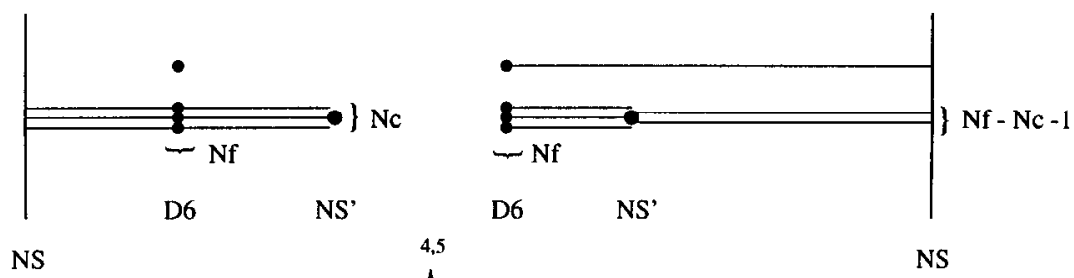

NS

(a)

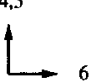

(b)

Fig. 8.

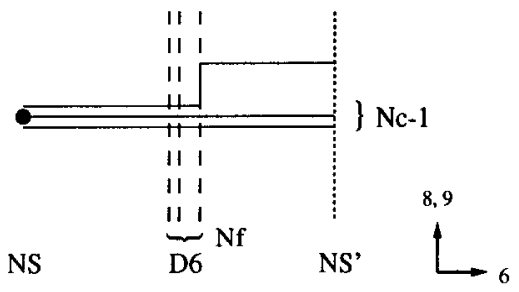

(a)

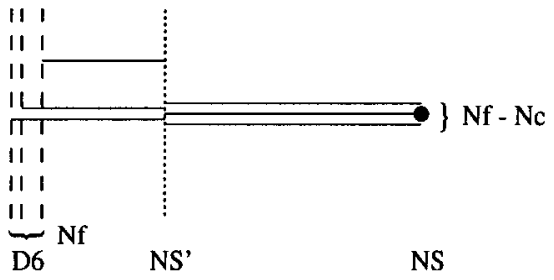

(b)

Fig. 9.

the world-volume theory and reduces the global symmetry to $U\left(N_{f}-1\right)$. The gauge symmetry in the electric theory remains unbroken.

The final configuration is the following. $N_{f}-N_{c}-1$ D4-branes stretched between NS and NS', $N_{f}-1$ D4-branes between the D6-branes and the $\mathrm{NS}^{\prime}$ brane, and a D4brane between one D6 and the NS brane separated from the other D4-branes in the $x^{4}, x^{5}$ directions (Fig. 8b). This corresponds to a mass term for one of the mesons (say $M_{\bar{N}_{f}}^{N_{f}}$ ) which, via the equations of motion, gives a vev to a magnetic quark. This reduces the gauge group and the global flavor symmetry. To obtain this configuration from the original configuration of the magnetic theory we have to connect a D4-brane between D6 and NS' with a D4-brane between NS and NS'. Only after this connection the resulting D4-brane can be lifted off from NS ${ }^{\prime}$ in the $x^{4}, x^{5}$ directions.

\section{Vevs for quarks}

The initial configuration is again one NS brane on the left side and one $\mathrm{NS}^{\prime}$ brane on the right, $N_{c}$ D4-branes stretched between NS and NS', all coincident and $N_{f}$ D6-branes with $x^{6}$ positions somewhere between the NS and the NS ${ }^{\prime}$ brane. If we tune the $x^{4}, x^{5}$ positions of one of the D6-branes such that it touches the D4-branes one (or more) of the D4-branes can split and the part between the D6 and the $\mathrm{NS}^{\prime}$ brane can move in the $x^{8}, x^{9}$ directions (Fig. 9a). We assume that one D4-brane splits on one specific D6-brane. If more than one D4-brane split on a specific D6-brane there would be more than one D4-brancs between NS and this specific D6. This corresponds to an $s$-configuration [4] and breaks supersymmetry which we want to avoid. The separation in $x^{8}, x^{9}$ corresponds to giving a vev to one of the quarks. Thus the gauge group in the electric theory is reduced to $U\left(N_{c}-1\right)$ and the number of massless (anti-) quarks is $N_{f}-1$.

The remaining $N_{c}-1$ D4-branes do not split and going through the usual steps 
we obtain the final configuration Fig. 9b. There are $N_{f}-N_{c}$ D4-branes between NS and $\mathrm{NS}^{\prime}$ and $N_{f}-1$ D4-branes between the D6-branes and the $\mathrm{NS}^{\prime}$ brane. The worldvolume theory on the D4-branes is $N=1$ supersymmetric gauge theory with gauge group $U\left(N_{f}-N_{c}\right), N_{f}-1$ magnetic quarks and mesons in the $\left(N_{f}-1, \overline{N_{f}-1}\right)$ representation. The fate of the split D4-brane deserves some comments. The piece that moved in the $x^{8}, x^{9}$ directions is stretched between one of the $N_{f}$ D6-branes and the $\mathrm{NS}^{\prime}$ brane and it corresponds to a meson which has been given a vev proportional to the distance to the other $N_{f}-1$ D4-branes in the $x^{8}, x^{9}$ directions (Fig. 9b). The other part which, in the initial position, is stretched between NS and one of the D6 disappears as the D6-branc moves through the NS branc and no additional D4-brane is created. In this "annihilation" process the number of D4-branes has to be reduced by one per D6brane. This annihilation process is just the reverse of the creation process of D4-branes, illustrated in Fig. 3.

We recall that giving a vev to the mesons still should leave us with $N_{f}^{2}$ massless mesons, some of them correspond to the Goldstone bosons associated with the breaking of the flavor symmetry. Moving one of the D4-branes in the magnetic picture along the $x^{8}, x^{9}$ directions corresponds to giving a vev to say $M_{1}^{1}$. Naively, it would seem as if we have now only $\left(N_{f}-1\right)^{2}$ massless mesons, since the strings between the D4-brane which we have moved and the rest of the $N_{f}-1$ D4-branes are all long. The resolution of this apparent paradox is that the mesons are really a mixture of D4-D4 and D4-D6 string states. (We recall, however, that there are no strings between D6 and D4 which terminate on each other.) The coefficients of this mixture depend on the particular point in the moduli space. At a generic point where all D4-branes and D6-branes are well separated the mesons are essentially D4-D4 strings. However, near the origin of the moduli space there must be a substantial component of the states coming from strings between D4- and D6-branes which do not terminate on each other.

We found that moving D6-branes or splitting D4-branes has an interpretation in field theory as mass terms or vevs for fields. Furthermore we were able to find a direct map between the deformations in the electric and magnetic theory which provides further evidence that the brane configuration of Fig. 2 reproduces Seiberg's duality. In the brane picture it is straightforward to see that the dual of the dual theory is the original one. All the steps have to be done in reversed order and we end up in the initial configuration. Recall that in field theory it is much more involved to show it [9].

\subsection{Adjoint duality}

In [10] dualities between theories with one chiral superfield in the adjoint representation was proposed. The electric theory contains an $U\left(N_{c}\right)$ vector multiplet, $N_{f}$ (anti)quarks $Q_{i}$ and $\tilde{Q}^{\tilde{i}}$ in the (anti-) fundamental representation of the gauge group and an adjoint $X$. Adding the following superpotential breaks $N=2$ to $N=1$ supersymmetry:

$$
W_{e}=\operatorname{Tr} \sum_{l=1}^{k+1} c_{l} X^{l}, \quad c_{k+1}=1 .
$$




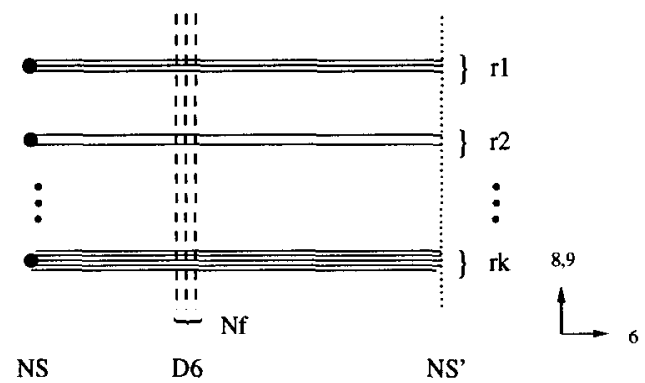

Fig. 10. A configuration with $k=3$.

The dual theory is an $U\left(k N_{f}-N_{c}\right)$ gauge theory with magnetic quarks $q^{i}$ and $\tilde{q}_{i}$, an adjoint field $Y$ and a set of $k$ mesons $M_{l}, l=1, \ldots, k$. These mesons correspond to the composite operators $Q X^{l-1} \tilde{Q}, l=1, \ldots, k$ in the electric theory. The magnetic superpotential has the form

$$
W_{m}=\operatorname{Tr} \sum_{l=1}^{k+1} d_{l} Y^{l}+\sum_{l=1}^{k} M_{l} \tilde{q} Y^{k-l} q, \quad d_{k+1}=1 .
$$

The exact map between the $c_{l}$ and $d_{l}$ has been worked out in the third reference of [10]. The electric superpotential has $k$ minima at $\partial W_{k} / \partial X=0$. This means that $X$ has $k$ different eigenvalues of multiplicity $r_{i}$ with $\sum_{i=1}^{k} r_{i}=N_{c}$ which breaks the gauge symmetry to $\prod_{i=1}^{k} U\left(r_{i}\right)$. In the following we assume that the adjoint is massive. We also note that there is never an adjoint flat direction due to the fact that the gauge group has an additional $U(1)$ factor. (For the $S U(N)$ gauge symmetry case a flat direction appears for even $N$ see, e.g., Ref. [19].)

A brane configuration representing this gauge theory was proposed in [7] and is the following. Instead of one there are $k$ NS branes whose $x^{8}, x^{9}$ positions correspond to the deformation parameters $c_{i}$ of the electric superpotential. Furthermore there is one NS' brane and the $i$ th NS brane is connected to the NS brane by $r_{i}$ D4-branes, and there are $N_{f}$ D6-branes which are needed to generate the matter (quark) content. Notice that there is no direct way to see the adjoint field $X$ directly as open strings, but there is a direct relation between the NS positions which are equal to the eigenvalues of the adjoint chiral superfield $X$ and the deformation parameters $c_{l}$ of the superpotential. The fact that we do not see $X$ as a fluctuation in the brane configuration is correlated with the observation that for a $U(N)$ gauge theory there is never an adjoint flat direction (which, if existed, would have had to correspond to some brane motion).

This configuration consists of $k$ copies of the configuration studied in the previous section and it is easy to guess what the final configuration corresponding to the magnetic theory must bc. There are $k$ sets of $N_{f}$ D4-branes stretched between the D6-branes and the $\mathrm{NS}^{\prime}$, and $k$ sets of $r_{i}$ D4-branes connecting the $i$ th NS with the $\mathrm{NS}^{\prime}$ brane. The resulting gauge group of the world-volume theory is broken: $U\left(k N_{f}-N_{c}\right) \rightarrow$ $\prod_{i=1}^{k} U\left(N_{f}-r_{i}\right)$ and there are $N_{f}$ magnetic (anti-) quarks in the fundamental of the broken gauge group. The $k$ mesons come from the $k$ sets of D4-branes between the 
D6-branes and the $\mathrm{NS}^{\prime}$. Again, near the origin of the moduli space appropriate mixing with D4-D6 string states occurs as discussed in the case of Seiberg's duality.

It presents no difficulty to realize the possible deformations in these theories: mass terms and vevs. It is completely analogous to the moves in the section on Seiberg's duality.

\section{Mass terms}

A mass term for one quark in the electric theory corresponds to lifting one of the $N_{f}$ D6-branes in the $x^{4}, x^{5}$ directions making the strings between the D4-branes and this D6-brane long and thus massive. The flavor symmetry is reduced to $S U\left(N_{f}-1\right)$ and the electric gauge group is unchanged.

The final configuration is the following. $k$ groups of $N_{f}-1$ D4-branes connecting the D6-branes to $\mathrm{NS}^{\prime}$ which are separated in the $x^{8}, x^{9}$ directions. There are $k$ groups of $N_{f}-r_{i}-1$ D4-branes connecting $\mathrm{NS}^{\prime}$ and the $\mathrm{NS}_{i}$ branes. The D6-brane which is lifted in the $x^{4}, x^{5}$ directions creates $k$ D4-branes stretched between this D6 and the $\mathrm{NS}_{i}$ branes. In the magnetic theory this corresponds to giving mass to the $\left(N_{f}, \overline{N_{f}}\right)$ component of the $k$ mesons. The equations of motion for the massive fields give a vev to one of the magnetic quarks. The magnetic gauge group is therefore $U\left(k N_{f}-N_{c}\right) \rightarrow$ $\prod_{i=1}^{k} U\left(N_{f}-1-r_{i}\right)$ and the flavor group is broken to $S U\left(N_{f}-1\right)$.

Vevs for quarks

We consider the case where one of the $r_{1}$ D4-branes connecting $\mathrm{NS}_{1}$ and $\mathrm{NS}^{\prime}$ splits into two parts separated in the $x^{8}, x^{9}$ directions. The gauge group is broken,

$$
U\left(r_{1}\right) \otimes U\left(r_{2}\right) \otimes \ldots \rightarrow U\left(r_{1}-1\right) \otimes U\left(r_{2}\right) \otimes \ldots
$$

and the flavor symmetry is reduced to $S U\left(N_{f}-1\right)$.

In the magnetic theory this corresponds to giving a vev to one component of one of the mesons. This also gives mass to one of the quarks due to the tree level superpotential and reduces the flavor group: $S U\left(N_{f}\right) \rightarrow S U\left(N_{f}-1\right)$. The magnetic gauge group remains unaffected. We would like to stress again that although we do not see the adjoint field, it is reassuring that the deformations associated with mesons containing the adjoint field are accounted for. This gives us confidence that the brane configuration suggested in [7] is indeed producing the "adjoint duality" [10].

\subsection{Duality of theories with product gauge groups}

In this section we want to study an extension of the brane configurations studied above. The brane configuration is drawn in Fig. 11a and consists of one NS brane, two $\mathrm{NS}^{\prime}$ branes and $N_{f}$ D6-branes between the NS and the first NS ${ }^{\prime}$ brane. In addition there are $N_{1}$ D4-branes stretched between the NS and the first $\mathrm{NS}^{\prime}$ brane, and $N_{2}$ D4-branes between the NS and the second $\mathrm{NS}^{\prime}$ brane. We take $N_{1} \geqslant N_{2}$ and chose the $x^{4}, x^{5}$ values of the two NS ${ }^{\prime}$ branes to coincide. The reason for these choices will become clear below. The world-volume theory on the D4-branes is a $N=1$ supersymmetry gauge theory with gauge group $U\left(N_{1}\right) \times U\left(N_{2}\right)$ with gauge couplings $1 / g_{1}^{2} \sim\left|x_{\mathrm{NS}_{1}^{\prime}}^{6}-x_{\mathrm{NS}}^{6}\right|$ and $1 / g_{2}^{2} \sim$ 


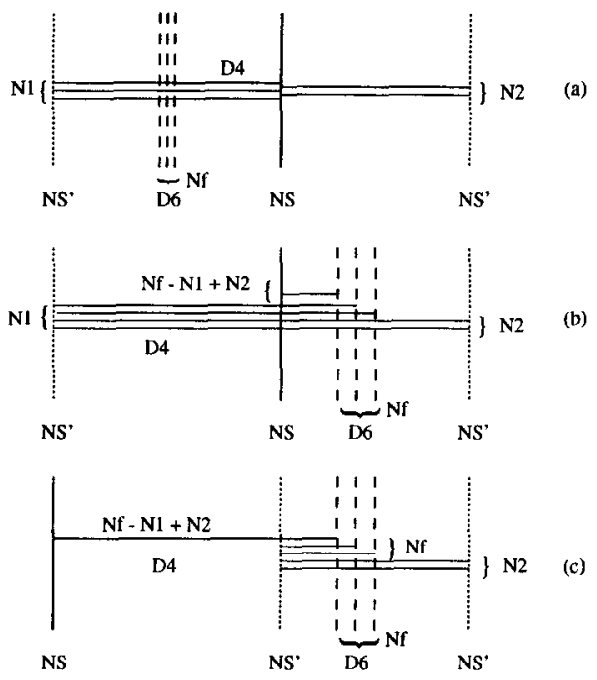

Fig. 11.

$\left|x_{\mathrm{NS}}^{6}-x_{\mathrm{NS}_{2}^{\prime}}^{6}\right|$. Since there are two independent gauge fields there are, at least classically, two $U(1)_{\mathcal{R}}$ symmetries. There are $N_{f}$ (anti-) quarks in the (anti-) fundamental, $Q$ and $\tilde{Q}$, and chiral multiplets, $N$ and $\tilde{N}$, in the $\left(N_{1}, \overline{N_{2}}\right)$ and the $\left(\overline{N_{1}}, N_{2}\right)$ representations of the gauge group. They arise from open strings between the two groups of D4-branes. The global symmetry of the world-volume theory is (recall that only the diagonal $S U\left(N_{f}\right)$ factor is manifest in the brane picture)

$$
S U\left(N_{f}\right) \times S U\left(N_{f}\right) \times U(1)_{J} \times U(1)_{B} \times U(1)_{\mathcal{R}}^{(1)} \times U(1)_{\mathcal{R}}^{(2)} .
$$

Of the $U(1)_{J}, U(1)_{\mathcal{R}}^{(1)}$ and $U(1)_{\mathcal{R}}^{(2)}$ symmetry only two combinations remain unbroken in the quantum theory and we will denote them by $U(1)_{\mathcal{R}}^{(1)}$ and $U(1)_{\mathcal{R}}^{(2)}$. The quantum numbers are determined by the requirement of gauge anomaly cancellation and are summarized in the following table:

$$
\begin{array}{lccccccc} 
& U\left(N_{1}\right) & U\left(N_{2}\right) & S U\left(N_{f}\right) & S U\left(N_{f}\right) & U(1)_{B} & U(1)_{\mathcal{R}}^{(1)} & U(1)_{\mathcal{R}}^{(2)} \\
W_{\alpha}^{(1)} & \left(N_{1}\right)^{2} & 1 & 1 & 1 & 0 & 1 & 1 \\
W_{\alpha}^{(2)} & 1 & \left(N_{2}\right)^{2} & 1 & 1 & 0 & 1 & 0 \\
N & N_{1} & \overline{N_{2}} & 1 & 1 & \frac{1}{N_{1}} & \frac{N_{1}-N_{2}}{N_{1}} & 1 \\
\tilde{N} & \overline{N_{1}} & N_{2} & 1 & 1 & -\frac{1}{N_{1}} & \frac{N_{1}-N_{2}}{N_{1}} & 1 \\
Q & N_{1} & 1 & N_{f} & 1 & \frac{1}{N_{1}} & \frac{N_{2}^{2}-N_{1}^{2}+N_{1} N_{f}}{N_{1} N_{f}} & \frac{N_{f}-N_{1}}{N_{f}} \\
\tilde{Q} & \overline{N_{1}} & 1 & 1 & \overline{N_{f}} & -\frac{1}{N_{1}} & \frac{N_{2}^{2}-N_{1}^{2}+N_{1} N_{f}}{N_{1} N_{f}} & \frac{N_{f}-N_{1}}{N_{f}}
\end{array}
$$

To find the dual of this theory we follow the same steps as in the case of Seiberg's duality. We begin by moving all D6-branes to the right of the NS brane and create, in the by now familiar manner, $N_{f}$ D4-branes stretched between the D6-branes and the NS brane. In the creation process each of these $N_{f}$ D4-branes inherits its $x^{4}, x^{5}$ positions 
from the D6-brane it ends on and its $x^{7}, x^{8}, x^{9}$ positions from the NS brane. We now connect the $N_{2}$ D4-branes on the right to $N_{2}$ out of the $N_{1}$ D4-branes on the left. By adjusting the $x^{4}, x^{5}$ positions of $N_{1}-N_{2}$ D6-branes, $N_{1}-N_{2}$ of the $N_{f}$ newly created D4-branes can be connected to those $N_{1}-N_{2}$ D4-branes stretched between the left NS' and NS brane, which had not yet been connected to the D4-branes on the right of the NS' brane (Fig. 11b). Note that this requires, in addition to $N_{1} \geqslant N_{2}$ also $N_{f} \geqslant N_{1}-N_{2}$.

Now the NS brane can be lifted in the $x^{7}$ direction which corresponds to turning on a FI term in the world-volume field theory. The NS brane can be moved to the left of the left NS' brane. At this stage nothing special happens and we can put the $x^{7}$ position of the NS branc back to its original value. The left NS' touches the D4-branes stretched between the NS and the other branes and they split. The final configuration is drawn in Fig. $11 \mathrm{c}$.

Let us summarize the field content of the D4 world-volume theory in the final brane configuration and discuss whether this is a possible $N=1$ duality by comparing the chiral rings of the two theories and checking the 't Hooft anomaly matching conditions. The theory has $U\left(N_{f}-N_{1}+N_{2}\right) \times U\left(N_{2}\right)$ gauge symmetry. Furthermore there are two kinds of quarks, magnetic (anti-) quarks $q$ and $\tilde{q}$ in the (anti-)fundamental of $U\left(N_{f}-N_{1}+N_{2}\right)$ which come from open strings between the two sets of D4-branes, and (anti-) quarks $r$ and $\tilde{r}$ in the (anti-)fundamental of $U\left(N_{2}\right)$ from strings between D4and D6-branes. There are also $N_{f}^{2}$ meson fields $M$, from open strings between the $N_{f}$ D4-branes terminating on the D6-branes, a chiral superfield $X$ in the adjoint of $U\left(N_{2}\right)$, whose scalar components are related to the fluctuations of the $N_{2}$ D4-branes between the two NS ${ }^{\prime}$ branes in the $x^{8}, x^{9}$ directions, and, finally, chiral superfields, $n$ and $\tilde{n}$ in mixed representations of the gauge group from strings between the left and the right set of D4-branes, both extending between one NS and one NS ${ }^{\prime}$ brane. The classical superpotential of the magnetic theory is

$$
W_{m}=q M \tilde{q}+n X \tilde{n}+n r \tilde{q}+\tilde{n} \tilde{r} q .
$$

The global symmetry of this theory is the same as for the original, electric theory. We summarize the quanlum numbers under the local and global symmetries as follows:

$\begin{array}{lccccccc} & U\left(\tilde{N}_{1}\right) & U\left(N_{2}\right) & S U\left(N_{f}\right) & S U\left(N_{f}\right) & U(1)_{B} & U(1)_{\mathcal{R}}^{(1)} & U(1)_{\mathcal{R}}^{(2)} \\ W_{\alpha}^{(1)} & \left(\tilde{N}_{1}\right)^{2} & 1 & 1 & 1 & 0 & 1 & 1 \\ W_{\alpha}^{(2)} & 1 & \left(N_{2}\right)^{2} & 1 & 1 & 0 & 1 & 0 \\ n & \tilde{N}_{1} & \overline{N_{2}} & 1 & 1 & \frac{1}{N_{1}} & \frac{N_{2}}{N_{1}} & 0 \\ \tilde{n} & \overline{N_{1}} & N_{2} & 1 & 1 & -\frac{1}{\tilde{N}_{1}} & \frac{N_{2}}{N_{1}} & 0 \\ q & \tilde{N}_{1} & 1 & \overline{N_{f}} & 1 & \frac{1}{\tilde{N}_{1}} & \frac{N_{1}^{2}-N_{2}^{2}}{N_{1}} & \frac{N_{1}}{N_{f}} \\ \tilde{q} & \overline{N_{1}} & 1 & 1 & N_{f} & -\frac{1}{\tilde{N}_{1}} & \frac{N_{1}^{2}-N_{2}^{2}}{N_{1} N_{f}} & \frac{N_{1}}{N_{f}} \\ r & 1 & N_{2} & N_{f} & 1 & 0 & \frac{N_{2}^{2}-N_{f} N_{2}-N_{1}^{2}+2 N_{f} N_{1}}{N_{f} N_{1}} & \frac{2 N_{f}-N_{1}}{N_{f}} \\ \tilde{r} & 1 & \overline{N_{2}} & 1 & \overline{N_{f}} & 0 & \frac{N_{2}^{2}-N_{f} N_{2}-N_{1}^{2}+2 N_{f} N_{1}}{N_{f} N_{1}} & \frac{2 N_{f}-N_{1}}{N_{f}} \\ M & 1 & 1 & N_{f} & \overline{N_{f}} & 0 & 2 \frac{N_{2}^{2}-N_{1}^{2}+N_{1} N_{f}}{N_{f} N_{1}} & 2 \frac{N_{f}-N_{1}}{N_{f}} \\ X & 1 & \left(N_{2}\right)^{2} & 1 & 1 & 0 & 2 \frac{N_{1}-N_{2}}{N_{1}} & 2\end{array}$


with

$$
\tilde{N}_{1}=N_{f}-N_{1}+N_{2}
$$

With these charge assignments all 't Hooft anomaly matching conditions for the global symmetries are satisfied.

To provide further evidence for the duality let us compare the gauge-invariant operators in the two theories. The mesons of the electric theory may all be identified with gauge singlets of the magnetic theory (in fact one has to consider $S U(N)$ gauge groups rather than $U(N))$ :

$$
M \sim Q \tilde{Q}, \quad r \sim Q \tilde{N}, \quad \tilde{r} \sim \tilde{Q} N, \quad X \sim N \tilde{N} .
$$

The relevant gauge invariant baryonic opcrators ${ }^{5}$ of the clcctric theory arc $(Q)^{N_{1}}$, $(\tilde{Q})^{N_{1}},(N)^{N_{2}}(Q)^{N_{1}-N_{2}}$ and $(\tilde{N})^{N_{2}}(\tilde{Q})^{N_{1}-N_{2}}$, and the corresponding operators of the magnetic theory are $(n)^{N_{2}}(q)^{\tilde{N}_{1}-N_{2}},(\tilde{n})^{N_{2}}(\tilde{q})^{\tilde{N}_{1}-N_{2}},(q)^{\tilde{N}_{1}}$ and $(\tilde{q})^{\tilde{N}_{1}}$, respectively. It is easy to see that the operators in the electric and their corresponding operators in the magnetic theory carry the same $U(1)$ charges and are in the same antisymmetric representation of the flavor group $S U\left(N_{f}\right)$ with dimensions $\left(\begin{array}{l}N_{f} \\ N_{1}\end{array}\right)=\left(\begin{array}{c}N_{f} \\ N_{f}-N_{1}\end{array}\right)$ and $\left(\begin{array}{c}N_{f} \\ N_{1}-N_{2}\end{array}\right)=\left(\begin{array}{c}N_{f} \\ N_{f}-N_{1}+N_{2}=\tilde{N}_{1}\end{array}\right)$ for the first two and the last two operators, respectively.

There is also a straightforward field theoretic interpretation of this duality. The starting point is $N=1$ supersymmetric QCD with gauge group $U\left(N_{1}\right)$ and $\tilde{N}_{f}=N_{f}+N_{2}$ (anti-) quarks. Now we can apply Seiberg's duality to this theory and we will find a magnetic theory with gauge group $U\left(\tilde{N}_{f}-N_{1}\right)=U\left(N_{f}+N_{2}-N_{1}\right), \tilde{N}_{f}=N_{f}+N_{2}$ magnetic (anti-) quarks and $\left(\tilde{N}_{f}\right)^{2}$ mesons. If we gauge the $U\left(N_{2}\right)$ subgroup of the $U\left(N_{f}+N_{2}\right)$ flavor group we obtain exactly the matter content of the electric and magnetic theories of our example. Actually the fields $M, X, r$ and $\tilde{r}$ are just the result of the decomposition of the original mesons and therefore there exist several relations between the $\mathcal{R}$ charges of the fields,

$$
\begin{aligned}
\mathcal{R}_{i}(M) & =2 \mathcal{R}_{i}(Q), & \mathcal{R}_{i}(X)=2 \mathcal{R}_{i}(N), \mathcal{R}_{i}(r)=\mathcal{R}_{i}(Q)+\mathcal{R}_{i}(N), \\
\mathcal{R}_{i}(q) & =1-\mathcal{R}_{i}(Q), & \mathcal{R}_{i}(n)=1-\mathcal{R}_{i}(N), \quad i=1,2,
\end{aligned}
$$

where the first line follows from the identification of the mesonic operators in the electric and magnetic theory (12), the second line follows from the first line and the fact that the superpotential (9) has $\mathcal{R}$ charge 2.

\section{Can brane configurations be related to non-supersymmetric gauge theories and dualities ?}

A natural question to ask after passing from theories with $N=2$ to those with $N=1$ is what are the brane setups that describe non-supersymmetric $4 \mathrm{D}$ gauge theories and in particular models with Seiberg-like dualities.

\footnotetext{
${ }^{5}$ For example, by $(Q)^{N_{1}}$ we mean $Q^{i_{1}} \ldots Q^{i_{N_{1}}}$ where contraction of the color indices with a totally antisymmetric rank $N_{1}$ tensor is understood.
} 
A priori it is not clear whether non-supersymmetric brane configurations represent consistent stable backgrounds. The stability of supersymmetric brane configurations is based on the no-force situation of static multiple brane solutions of the low-energy effective action of type IIA and type IIB string theories [14-16]. This is lost when supersymmetry is completely broken by the brane background configuration; the BPS condition is then no longer satisfied.

We thus have to expect that the brane configurations we are about to discuss are unstable. Note that also the stability of the configurations considered in [7] should be carefully re-examined, as there is a unbalanced force on the NS branes from the D-branes stretched between them [21].

In what follows we will ignore this apparent problem and consider these configurations. We will apply the same moves that were used in the supersymmetric cases and compare the matter content looking for cases in which 't Hooft anomaly matching conditions are satisfied. In those cases in which they are satisfied we may have candidates for dual pairs. (Thus, if at all, the justification for looking at these configurations is a posteriori.)

In the following table we list several brane configurations which break all supersymmetries. The table includes only branes which are along some coordinate axis and not branes at angles. We denote the $\mathrm{NS}^{(1)}, \mathrm{NS}^{(2)}$ and D6-branes by their world volume coordinates which they have in addition to $x^{0}, x^{1}, x^{2}, x^{3}$. The table also includes possible $U(1)$ symmetries that have their origin in a rotation invariance in a subspace of $10 \mathrm{~d}$ space-time.

$\begin{array}{lccccc} & \mathrm{NS}^{(1)} & \mathrm{NS}^{(2)} & \mathrm{D} 6 & U(1) & \mathrm{D} 4 \text { created } \\ \text { (i) } & x^{4}, x^{5} & x^{4}, x^{7} & - & (89) & - \\ \text { (ii) } & x^{4}, x^{5} & x^{4}, x^{5} & x^{4}, x^{8}, x^{9} & (89) & \text { no } \\ \text { (iii) } & x^{4}, x^{5} & x^{8}, x^{9} & x^{4}, x^{5}, x^{8} & (45) & \text { no } \\ \text { (iv) } & x^{4}, x^{5} & x^{8}, x^{9} & x^{4}, x^{7}, x^{8} & \text { no } & \text { no } \\ \text { (v) } & x^{4}, x^{5} & x^{4}, x^{7} & x^{7}, x^{8}, x^{9} & (89) & \text { yes } \\ \text { (vi) } & x^{4}, x^{5} & x^{4}, x^{7} & x^{4}, x^{5}, x^{7} & (89) & \text { no } \\ \text { (vii) } x^{4}, x^{5} & x^{4}, x^{7} & x^{5}, x^{7}, x^{8} & \text { no } & \text { no }\end{array}$

Each item describes one example out of a family of similar configurations. For instance $\mathrm{NS}^{(2)}$ in (i) can be any of the following possibilities. $x^{i}, x^{j}$ with $i \in\{4,5\}$ and $j \in\{7,8,9\}$.

It is now straightforward to realize that indeed all the supersymmetries are broken for the setups of the table. Let us demonstrate it for the cases that $\mathrm{NS}^{(2)}$ is $x^{4}, x^{7}$. The supersymmetry parameters have to obey

$$
\epsilon_{L}=\Gamma^{0} \ldots \Gamma^{5} \epsilon_{L} ; \quad \epsilon_{R}=\Gamma^{0} \ldots \Gamma^{5} \epsilon_{R}
$$

due to the NS brane and

$$
\epsilon_{L}=\Gamma^{0} \ldots \Gamma^{4} \Gamma^{7} \epsilon_{L} ; \quad \epsilon_{R}=\Gamma^{0} \ldots \Gamma^{4} \Gamma^{7} \epsilon_{R}
$$

due to the NS" brane. Substitute $\epsilon_{L}$ from (15) into (16) to get 


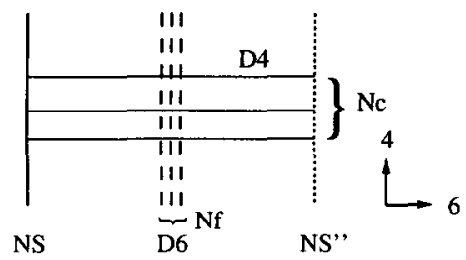

(a)

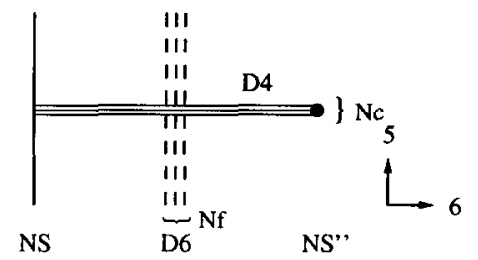

(b)

Fig. 12.

$$
\epsilon_{L}=-\Gamma^{5} \Gamma^{7} \epsilon_{L}=\Gamma^{5} \Gamma^{7} \Gamma^{5} \Gamma^{7} \epsilon_{L}=-\epsilon_{L}
$$

which obviously means that there is no non-trivial solution for $\epsilon_{L}$ and similarly for $\epsilon_{R}$.

The failure to have supersymmetric charges may imply that also adjoint fermions associated with strings between the D4 branes cannot exist. To demonstrate Seiberg's duality in an $N=0$ field theory model, we assume in the next subsection that the latter does not hold. The examination of this assumption, and the analysis of $N=0$ models deserve further investigation.

Seiberg's duality followed in the supersymmetric models from brane configurations that have D6-branes that, when moving them along the $x^{6}$ direction past the NS5-brane, necessarily meet the NS5-branein space-time. For this it is necessary that the NS and the D6-branes have no common transverse direction except $x^{6}$. Only then do we create new D4-branes. The last column of the table indicates whether the model has this property.

\subsection{Seiberg's duality in $N=0$}

As indicated in the table model (v) can incorporate non-trivial NS brane rearrangements. The basic setup for this case, which is described in Fig. 12, is related to the configuration of Fig. 1, with an NS brane along $x^{4}, x^{7}$, denoted by $\mathrm{NS}^{\prime \prime}$, replacing the left side NS.

We want to emphasize again the issue of the stability of the brane configuration. Bcing aware of potential problems, we nevertheless proceed in the same way as we did when considering supersymmetric configurations.

The fermionic content of the field theory is summarized in the following table:

$\begin{array}{lccccc} & U\left(N_{c}\right) & S U\left(N_{f}\right) & S U\left(N_{f}\right) & U(1)_{B} & U(1)_{R} \\ \psi_{Q} & N_{c} & N_{f} & 1 & \frac{1}{N_{c}} & \frac{-N_{c}}{N_{f}} \\ \psi_{\tilde{Q}} & \bar{N}_{c} & 1 & \bar{N}_{f} & \frac{-1}{N_{c}} & \frac{-N_{c}}{N_{f}} \\ (\lambda / \psi)_{\mathrm{el}} & N_{c}^{2} & 1 & 1 & 0 & 1\end{array}$

where the massless fermions were classified according to the maximal global symmetry given in the first line. $U(1)_{R}$ is the axial symmetry unbroken by instantons. $\psi_{Q}$ and $\psi_{\tilde{Q}}$, the ordinary quarks and anti-quarks, are associated with the 4-6 strings, and $\lambda_{\mathrm{el}}$ are the "gauginos".

Actually there are two adjoint fermions, the gauginos that originate from $W_{\alpha}$ and a second adjoint fermion $\psi_{a d j}$ that is associated with $\Phi$. Note that in the present model 
there is a real adjoint scalar field related to the motion of the D4 along $x^{4}$ whereas its $N=2$ companion, the second real scalar field related to the motion of the D4 along $x^{5}$, is absent. The reason that we have included only one adjoint fermion is that there is only one $U(1)_{R}$ symmetry unbroken by instantons, since the charges of $\lambda$ and $\psi$ are not independent due to a Yukawa term. Therefore, there is only one linear combination of the fermions, denoted by $(\lambda / \psi)_{\mathrm{el}}$ that remains massless. Note also that in nonsupersymmetric field theories chiral symmetry protects the masslessness of fermions and not of their associated "scalar partners".

Performing a sequence of moves that is identical to the one performed for the $N=1$ case we cnd up with a branc configuration similar to the one described in Fig. 4, where again $\mathrm{NS}^{\prime \prime}$ replaces $\mathrm{NS}^{\prime}$. Using the same rules to convert the branes and the strings between them to fields on the 4D world-volume as for the electric theory one now finds the following fields:

$\begin{array}{lccccc} & U\left(N_{f}-N_{c}\right) & S U\left(N_{f}\right) & S U\left(N_{f}\right) & U(1)_{B} & U(1)_{R} \\ \psi_{q} & N_{f}-N_{c} & \overline{N_{f}} & 1 & \frac{1}{N_{f}-N_{c}} & \frac{N_{c}}{N_{f}}-1 \\ \psi_{\tilde{q}} & \overline{N_{f}-N_{c}} & 1 & N_{f} & -\frac{1}{N_{f}-N_{c}} & \frac{N_{c}}{N_{f}}-1 \\ \lambda_{\text {mag }} & \left(N_{f}-N_{c}\right)^{2} & 1 & 1 & 0 & 1 \\ \psi_{M} & 1 & N_{f} & \bar{N}_{f} & 0 & -2 \frac{N_{c}}{N_{f}}+1\end{array}$

The emergence of the magnetic quarks, anti-quarks and "magnetic gaugino" from strings is similar to that in $N=1$ theory. The mesons, as in the $N=1$ cases, are again due to strings among the $N_{f}$ D4-branes stretched between the D6-branes and the NS" branes. However, now the D4-brane can move only along one direction, $x^{7}$. Following our dictionary this implies real and not complex $U\left(N_{f}\right)$ real scalar fields. In terms of the field theory mesons are composites of $\phi_{M} \sim \psi_{Q} \psi_{\tilde{Q}}$ whereas the mesonic fermions $\psi_{M}$ are composites of $\psi_{M} \sim \psi_{Q} \lambda \psi_{\bar{Q}}$ with $U(1)_{R}$ charge $-2 \frac{N_{c}}{N_{f}}+1$. The masslessness of these fermions is protected by $U(1)_{R}$ symmetry. In fact without any additional term in its action the magnetic theory has an additional $U(1)$ symmetry which does not have a counterpart in the electric one. This is obviously avoided by the introduction of a Yukawa term which is the remnant of the $M Q \tilde{Q}$ superpotential term in the $N=1$ magnetic theory.

It is now straightforward to check that the 't Hooft anomalies associated with $S U\left(N_{f}\right)^{3}, S U\left(N_{f}\right)^{2} \times U_{B}(1), S U\left(N_{f}\right)^{2} U(1)_{R}, U(1)_{R}\left(U(1)_{R}\right)^{3},\left(U(1)_{R}\right)^{2} U(1)_{B}$, $\left(U(1)_{R}\right)^{2} U(1)_{B}, U(1)_{B}^{3}$ of the electric and magnetic theories match.

In Section 3.2 certain moves of brane configurations led to a duality between $U\left(N_{c}\right)$ and $U\left(k N_{f}-N_{c}\right)$ gauge theories where $k$ was the number of NS branes taken instead of the basic configuration with one NS brane. Repeating the same structure with $\mathrm{NS}^{\prime \prime}$ replacing the $\mathrm{NS}^{\prime}$ brane establishes a similar non-supersymmetric duality.

The magnetic theory includes now a set of $k$ composite mesons $\psi_{M} \sim \psi_{Q}(\lambda)^{2 l-1} \psi_{\bar{Q}}$ with $l=1, \ldots, k$. Their $U(1)_{R}$ charges are $-2\left(N_{c} / N_{f}\right)+2 l-1$. The sum of the $U(1)_{R}$ charges and the sum of their cubes are $-N_{c}^{2}-1$ and $-\left(2 N_{c}^{4} / N_{f}^{2}\right)+N_{c}^{2}-1$, respectively and are identical to the corresponding anomaly factors in the electric theory. 
Several remarks are now in order:

(i) The original supersymmetric duality was supported by the following tests: 't Hooft anomaly equations, chiral rings, mass and vev deformations. In the non-supersymmetric analogs only the first test is available. Moreover, recall that 't Hooft anomaly matching conditions are only necessary conditions to maintain chiral symmetry but by no means sufficient ones.

(ii) The $N=1$ magnetic theory is characterized by a moduli space parametrized by expectation values $\langle q\rangle,\langle\tilde{q}\rangle$ and $\langle M\rangle$. The non-supersymmetric theory, where the dual squarks and mesons are massive, has a unique minimum of the potential at the origin of the moduli space $\langle q\rangle=\langle\tilde{q}\rangle=\langle M\rangle=0[17]$.

(iii) Seiberg's duality was studied in the context of softly broken supersymmetric QCD in [17]. It was shown there that indeed for the case that $R$ is not broken and the "gaugino" remains massless one can justify the duality, at least for small squark masses. However, QCD inequalities seem to forbid massless fermionic mesons in the decoupling limit of large squark masses. In the case of no massless adjoint fermion one can show that there are no non-trivial solution to 't Hooft anomaly equations and thus there is no such duality. Notice, that the model (v) has, at least classically a $U(1)_{R}$ symmetry.

(iv) The setup with NS and $\mathrm{NS}^{\prime \prime}$ can be achieved from that with NS and $\mathrm{NS}^{\prime}$ by performing a complex rotation of $z \rightarrow e^{i \theta} z w \rightarrow e^{i \theta} w$ with $z=x^{8}+i x^{4}$ and $w=x^{9}+i x^{7}$ similar to the one suggested in [20]. The construction of explicit soft susy breaking terms is currently under investigation.

(v) As we have emphasized at the beginning of this section it is difficult to sce how the non-supersymmetric configurations could be consistent string backgrounds. If indeed they are unstable and one can argue that any gauge theory that admits Seiberg's duality has to have a brane counterpart, this may indicate that the $U(1)_{R}$ of the $R$ model discussed in [17] is broken for any squark mass. Conversely, if one finds that there is a region where $U(1)_{R}$ is unbroken, it might be a hint that there should be a stable brane configuration describing it.

\section{Instantons and brane configurations}

The brane configurations described in the previous sections correspond to the perturbative regime of certain supersymmetric and non-supersymmetric 4D gauge field theories. We now want to explore the possibility of translating non-perturbative gauge fields, namely instantons, into brane configurations. In [4] Euclidean D1 branes were introduced in the type IIB theory to describe 3D instantons. This was further explored in Ref. [5,6].

The branes that we have in our arsenal to induce 4D instanton effects are the following objects in their Euclidean formulation: even branes of IIA and the NS5-branes.

Let us identify now the conditions one has to impose on type IIA branes describing 4D instantons. ${ }^{6}$

\footnotetext{
${ }^{6}$ We thank $\mathrm{O}$. Aharony for pointing out to us the identification of the D0-branes with zero-size instantons.
} 
(i) To describe a point in the 4D world volume of the D4-branes, the directions $x^{0}, x^{1}, x^{2}, x^{3}$ have to be transverse directions to the brane.

(ii) Since in 4D gauge theories instantons break $1 / 2$ of the supersymmetries, the additional brane should break the $N=2$ theory down to $N=1$, and break supersymmetry all together in the $N=1$ theory.

(iii) The branes added should "communicate" with the D4-branes. In general this can be achieved if they end on the D4-branes, or if there are open strings stretched between them and the D4-branes.

(iv) The action of the instantons should be finite and be proportional to $\frac{1}{g^{2}}$.

(v) There should not be any (non-singular) instanton effect in the branc sctup that corresponds to the abelian theory.

From (i) it follows that one can add Euclidean D0, D2, D4-branes with world volumes embedded in the $\left\{x^{4}, x^{5}, x^{6}, x^{7}, x^{8}, x^{9}\right\}$ subspace of $\mathbb{R}^{1,9}$. If we demand that the new branes will end on the NS5-branes or the D4-branes, that will leave only D0- and D2branes and Euclidean NS strings. Checking the Euclidean D2-branes one finds that the branes along $x^{4}, x^{5}, x^{6}$ and $x^{6}, x^{8}, x^{9}$ break supersymmetry altogether in the $N=2$ case whereas the branes with $x^{4}, x^{6}, x^{i}$ (or $x^{5}, x^{6}, x^{i}$ ) where $i \in\{7,8,9\}$ have the required supersymmetry breaking pattern. Recall that a Euclidean D-brane imposes a condition on the parameter of supersymmetry transformation that differs by a factor of $i$ for the one imposed by an ordinary Minkowski brane. For instance the $x^{4}, x^{5}, x^{6}$ brane requires that $\epsilon_{L}=i \Gamma^{4} \Gamma^{5} \Gamma^{6} \epsilon_{R}$. The problem with the Euclidean D2-branes is that their action, which is proportional to their volume, always involves one non-compact direction and thus is infinite.

We are left with the D0-branes stretched along $x^{6}$ between the NS branes. Their action is proportional to $\left|x_{\mathrm{NS}_{1}}^{6}-x_{\mathrm{NS}_{2}}^{6}\right|=\lambda_{\text {string }} / g^{2}$, with $\lambda_{\text {string }}$ being the string coupling constant. Since there is an extra $\frac{1}{\lambda_{\text {string }}}$ factor associated with any D-brane action, the D0 action is proportional to $1 / g^{2}$. The D0-branes affect the D4-branes via the open strings that are stretched between them. At first sight they seem to violate condition (v) since they can exist also in case of single D4-brane. In fact our claim is that they correspond to zero-size instantons which can show up also in the abelian theory. This agrees also with arguments given in [18] which identify $\mathrm{D}(p-4)$-branes in $\mathrm{D} p$-branes as zero-size instantons. Finite size instantons are associated with the Higgs phase on their world line. The brane picture of the phase transition from zero to finite size instantons, as well as questions like how is the $U(1)_{R}^{45}$ symmetry broken by fermionic zero-modes are under current investigation. It is interesting to note, that the D0-branes can be obtained from the 3D type IIB setup by compactifying $x^{3}$ on a circle on which D1-brane is wrapped and performing $T$-duality along $x^{3}$. This is the same duality needed to pass from the IIB brane configurations associated with the $3 \mathrm{D}$ physics to the type IIA brane configurations of [7]. 


\section{Discussion}

String backgrounds that include brane configurations can be related to the $4 \mathrm{D}$ field theory that describes the low energy phase of nature in the following ways: (i) The universe may be associated with a D3-brane (or a truncated D4) which is part of the string background. (ii) Physics on branes may shed certain new light on field theories even without the scenario mentioned in (i). The recent progress in the interplay between brane physics and field theory that originated in [4] belongs to (ii). It should be interesting to explore also option (i).

In the present paper we have unraveled an additional piece in the puzzle of the full relations between field and brane theories. We elaborate on the brane rearrangements that manifest the equivalence between the Higgs branches of "dual" $N=2$ models. We provided further evidence for the construction of [7] by analyzing the correspondence between the mass and vev deformations of the $N=1$ electric and magnetic theories. This was done for the setup that translates to the original duality of Seiberg [9] as well as to the model that includes adjoint chiral multiplet [10]. We also suggested a new construction that corresponds to a Seiberg duality in field theories with product gauge groups. A great challenge in the interplay between string backgrounds and 4D field theory is the case without supersymmetry. Unfortunately, we were not able to suggest a mechanism that stabilizes the brane constructions we have considered. However, by closing our eyes to this problem we discussed a model that may be related to a duality of softly broken supersymmetric QCD model with unbroken $U(1)_{R}$ symmetry. Lastly, we enlist certain conditions that we found to be plausible in the search for branes that may mimic gauge instantons. We have elaborated on the possible identification of the instantons within the brane picture. It seems to us that D0-branes embedded in a D4-brane may correspond to a zero-size instanton.

Certainly the journey toward a full understanding of the interplay between brane configurations of type II string theories and gauge field theories in 3D and 4D is still in its infancy and there are many open questions to address. Let us mention several of them related to the present work.

(i) The constructions of Refs. [4,7] will be proven to be more powerful if novel dualities are discovered. Generalizing the way we have built the model with product groups should lead to novel field theory dual pairs.

(ii) A proper understanding of brane instantons might enable us to explore, directly in the brane picture, field theoretic instanton phenomena such as their contribution to the complex coupling $\tau$ in $N=2$ theories, the superpotential of $N=1$ theories with $N_{C}>N_{f}$, the brcaking of the axial $U(1)\left(U(1)_{45}\right)$, etc. In fact, by considering other possible brane attachments not obeying the conditions of Section 6, one may identify additional non-perturbative field theory phenomena. This immediately raises the more general question of the relation between the space of 4D field theories and the space of consistent brane configurations.

(iii) In this context one should also raisc the question of stability of the brane configurations and whether it is necessary for deriving field theory results. 
(iv) Recently, the brane setup for $N=2$ was analyzed, yielding explicit solutions for the Coulomb branch of a large family of four-dimensional $N=2$ field theories with zero, negative or positive beta function [21]. There are numerous questions that one can address using those new results in relation to the topics discussed in our work.

\section{Acknowledgements}

We would like to thank $\mathrm{O}$. Aharony, D. Ghoshal and D. Kutasov for useful conversations.

\section{References}

[1] S. Kachru, A. Klemm, W. Lerche, P. Mayr and C. Vafa, Nucl. Phys. B 459 (1996) 537, hep-th/9508155; A. Klemm, W. Lerche, P. Mayr, C. Vafa and N. Warner, Nucl. Phys. B 477 (1996) 746, hep-th/9604034.

[2] S. Katz, A. Klemm and C. Vafa, Geometric Engineering of Quantum Field Theories, hep-th/9609239; S. Katz and C. Vafa, Geometric Engineering of $N=1$ Quantum Field Theories, hep-th/961 1090.

[3] M. Bershadsky, A. Johansen, T. Pantev, V. Sadov and C. Vafa, F-theory, Geometric Engineering and $N=1$ Dualities, hep-th/9612052;

C. Vafa and B. Zwiebach, $N=1$ Dualities of $S O$ and $U S p$ Gauge Theories and T-Duality of String, hep-th/9701015.

[4] A. Hanany and E. Witten, Type IIB superstrings, BPS monopoles, and three-dimensional gauge dynamics, hep-th/9611230.

[5] J. de Boer, K. Hori, Y. Oz and Z. Yin, Branes and Mirror Symmetry in $N=2$ Supersymmetric Gauge Theories in Three Dimensions, hep-th/9702154.

[6] J. de Boer, K. Hori, H. Ooguri and Y. Oz, Mirror symmetry in three-dimensional theories, $S L(2, \mathbb{Z})$ and D-brane moduli spaces, hep-th/9612131; Mirror symmetry in three-dimensional gauge theories, quivers and D-branes, hep-th/961 1063.

[7] S. Elitzur, A. Giveon and D. Kutasov, Branes and $N=1$ in String Theory, hep-th/9702014.

[8] N. Evans, C.V. Johnson and A. Shapere, Orientifolds, Branes, and Duality of 4D Gauge Theories, hep-th/9703210.

[9] N. Seiberg, Nucl. Phys. B 435 (1995) 129, hep-th/9411149.

[10] D. Kutasov, Phys. Lett. B 351 (1995) 230, hep-th/9503086;

D. Kutasov and A. Schwimmer, Phys. Lett. B 354 (1995) 315, hep-th/9505004;

D. Kutasov, A. Schwimmer and N. Seiberg, Nucl. Phys. B 459 (1996) 455, hep-th/9510222.

[11] A. Strominger, Phys. Lett. B 383 (1996) 44, hep-th/9512059.

$[12] \mathrm{H}$. Ooguri and C. Vafa, Geometry of $N=1$ Dualities in Four Dimensions, hep-th/9702180.

[13] I. Antoniadis and B. Pioline, Higgs branch, hyperkähler quotient and duality in SUSY $N=2$ Yang-Mills theories, hep-th/9607058.

[14] E. Bergshoeff, M. de Roo, E. Eyras, B. Janssen and J.P. van der Schaar, Multiple intersections of D branes and $\mathrm{M}$ branes, hep-th/9612095.

[15] R. Argurio, F. Englert and L. Houart, Intersection Rules for $p$-Branes, hep-th/9701042.

[16] I.Ya. Arefeva, K.S. Viswanathan, A.I. Volovich and I.V. Volovich, Composite $p$-Branes in Various Dimensions, hep-th/9701092;

I. Aref'eva and A. Volovich, Composite p-Branes in Diverse Dimensions, hep-th/9611026.

[17] O. Aharony, M.E. Peskin, J. Sonnenschein and S. Yankielowicz, Phys. Rev. D 52 (1995) 6157, hepth/9503186.

[18] E. Witten, Nucl. Phys. B 460 (1996) 541;

M. Douglas, Branes within Branes, hep-th/9512077.

[19] O. Aharony, J. Sonnenschein and S. Yankielowicz, Nucl. Phys. B 449 (1995) 509, hep-th/9504113.

[20] J.L.F. Barbón, Rotated Branes and $N=1$ Duality, hep-th/9703051.

[21] E. Witten, Solutions of Four-Dimensional Field Theories via $M$ Theory, hep-th/9703166. 